\title{
MIGRAÇÃO, VULNERABILIDADE E (IN) JUSTIÇA AMBIENTAL: DESAFIOS E PERPECTIVAS
}

\author{
Raquel Fabiana Sparemberger ${ }^{1}$ \\ Vanessa Vergani ${ }^{2}$
}

\section{RESUMO}

A degradação ambiental atinge níveis alarmantes e a destruição de ecossistemas vêm provocando a migração forçada de pessoas atingidas por catástrofes ambientais em todo mundo. As formas e as conseqüências dessas agressões ao meio ambiente assumiram, na contemporaneidade, níveis inquietantes, principalmente pela atuação dos países industrializados e da sociedade, grandes produtores de riscos ecológicos, tornando pessoas vulneráveis e acarretando o que se pode chamar de (in) justiça ambiental.

Palavras-chave: Degradação. Injustiça. Migração. Vulnerabilidade.

\section{INTRODUÇÃO}

Os desastres ambientais e os riscos de desastres sejam eles de origens naturais ou decorrentes da ação do homem se apresentam como um dos grandes temas do direito ambiental contemporâneo, no que diz respeito ao agravamento dos riscos decorrentes do desenvolvimento tecnológico e, principalmente devido às conseqüências humanitárias advindas desses fenômenos.

Os riscos ambientais não são eqüitativamente distribuídos, o que preconiza o movimento da justiça ambiental, e fatores como a pobreza associados a vulnerabilidade de populações e comunidades inteiras estão no centro da distribuição destes riscos. Devido a isso, pode-se constatar que a exposição de pessoas vulneráveis aos riscos de desastres ecológicos contribuem para uma maior exposição à violação dos direitos humanos.

Diante dessa nova problemática global ambiental onde as transformações no meio ambiente em forma de risco de desastres ambientais condicionam as incertezas fabricadas por esta sociedade atual e que coloca em ameaça um contingente de pessoas que necessitam se deslocar em virtude do risco de desastre ou o desastre ambiental propriamente dito.

A migração, seja ela permanente ou temporária, tem sido sempre uma tradicional resposta ou estratégia de sobrevivência das pessoas ou populações que se confrontam com essa perspectiva, impacto ou conseqüência dos desastres ambientais. A grande complexidade dos desastres ambientais e seus riscos são causados pela interação entre fatores sociais e econômicos no meio ambiente, portanto, exacerbando a vulnerabilidade das pessoas e populações inteiras, intensificando os seus impactos quando se efetivam ou simplesmente ameaçam sua ocorrência. 
A complexidade das causas que originam o deslocamento da população não torna unânime a definição de uma expressão única que designe os movimentos populacionais relacionados à questões ambientais. Excluindo-se as catástrofes ambientais, em que os deslocamentos são forçados e inevitáveis por não haver quaisquer condições de sobrevivência no local atingido, a grande maioria dos casos restantes pode apresentar múltiplas variáveis que, somadas, resultam na decisão de deslocar-se.

Em muitos casos, razões econômicas e sociais, como a pobreza, o desemprego, a plena falta de perspectivas, somam-se às causas ambientais para produzir os deslocamentos, ou ainda, são resultados diretos da insuficiência de recursos naturais em determinadas regiões, sendo impossível atribuir a uma ou outra causa em especial aquela que tenha sido determinante para provocar a movimentação.

A decisão de migrar, seja entre os limites nacionais ou além das fronteiras de seus Estados, é uma das mais importantes estratégias de sobrevivência adotada pelas pessoas ou comunidades inteiras frente aos desastres ambientais, sejam naturais ou causados pelo homem, ou ainda diante do risco de desastre ecológico. Contudo, a relação envolvendo as mudanças ambientais, como os riscos de desastres ambientais, são a causa e a conseqüência das migrações decorrentes dos mesmos.

Em um contexto onde o problema do meio ambiente se dá de uma forma global e a degradação ambiental tem acelerado o número de indivíduos e populações que estão vendo a migração como uma opção. Por isso, há a necessidade de estudo e pesquisa acerca dessa temática, principalmente no que diz respeito às dimensões humanitárias internacionais das migrações devido a desastres e riscos ambientais.

O problema dessas pessoas ou populações, classificadas neste trabalho como migrantes ambientais, que compreendem aqueles indivíduos que se deslocam dentro de seus próprios países, ou internacionalmente, fugindo em função da ação de acontecimentos naturais ou provocados pelo homem (como o desenvolvimento de projetos de infra-estrutura, que incluem a construção de usinas hidrelétricas, barragens, estradas, ferrovias, projetos de irrigação, etc.) ou o risco de desastres, mesmo influenciados por causas que, embora sejam decorrentes de fatores ambientais, ainda permitam a permanência no local.

Diante disso, a migração decorrente de causas ambientais, mas que englobam ainda fatores sociais e econômicos deverá ser tratada não apenas no campo dos direitos humanos, mas também pelo direito ambiental internacional, pois se necessita a garantia a todas as pessoas que possam a vir perder seus lares devido a desastres ambientais ou riscos ambientais. 


\section{VULNERABILIDADE AMBIENTAL ANTE OS RISCOS DE DESASTRES ECOLÓGICOS}

Os problemas ambientais, por sua própria natureza e pelo contexto social e econômico, têm conseqüências ainda mais graves à realização dos direitos dos grupos vulneráveis, incluindo as mulheres, crianças, idosos, pobres entre outros. É preciso ficar claro que os meios de proteção podem voltar-se à garantia tanto dos direitos que são inerentes a todos os seres humanos em virtude de sua própria existência, assim como dos direitos atinentes a determinadas condições sociais.

Como ensina Trindade, há direitos que são essencialmente individuais, que podem ser protegidos somente no próprio indivíduo, mas há outros que podem ser mais bem protegidos através de um grupo, particularmente no caso de vir este grupo a ser vitimado. ${ }^{3}$

Cabe ressaltar a afirmativa do sociólogo Ulrich Beck de que a degradação ou poluição ambiental possui uma dimensão democrática, no sentido de que afeta todas as pessoas indistintamente, independente da classe social que integram, há sim indivíduos e grupos sociais mais vulneráveis aos efeitos negativos da degradação ambiental. ${ }^{4}$ Assim, Beck reconhece tal questão e refere que determinados grupos sociais, em razão do seu baixo poder aquisitivo, encontram-se mais vulneráveis a certos aspectos da degradação ambiental, de tal sorte que os riscos se acumulam abaixo, na medida em que as riquezas se acumulam acima. ${ }^{5}$

De toda forma, a degradação do meio ambiente provoca o desastre ambiental, que tem como característica principal a sua dimensão coletiva, pois pode ser entendido a partir de diferentes perspectivas, sejam elas sociais, ambientais, econômicas, entre outras. Neste sentido, o desastre ecológico pode ter como causa estritamente humana, decorrente do desenvolvimento de atividades e tecnologias que envolvem certo nível de risco ambiental, ou ser o produto de fenômenos naturais, nos quais também incidem a ação humana, como exemplo o aquecimento global, em grande parte provocada por fatores humanos. ${ }^{6}$

Laura Westra classifica, tradicionalmente, os desastres naturais em três categorias, quais sejam: a) evolução em longo prazo da degradação ambiental, incluindo o aquecimento global, erosão do solo, desertificação, desmatamento; b) imediatos desastres naturais, incluindo terremotos, secas, furacões, tornados e erupções vulcânicas; c) acidentes, incluindo os industriais e os desastres químicos. ${ }^{7}$

Ainda, o IASC - Inter-Agency Standing Comitee ${ }^{8}$ em seu manual designado "Direitos Humanos e Desastres Naturais linhas diretrizes e operacionais e manual sobre o respeito aos direitos humanos em situações de desastres naturais", trazem os desastres naturais como conseqüências de eventos decorrentes de perigos naturais que ultrapassam a capacidade local de resposta e afetam seriamente o desenvolvimento econômico e social de uma região, gerando perdas humanas, econômicas e ambientais. Tradicionalmente, desastres naturais estão sendo vistos como situações que criam desafios e problemas, principalmente no que diz respeito aos direitos humanos. Contudo, deve ser reconhecido que a proteção aos direitos humanos também precisa ser concedido neste contexto. ${ }^{9}$ 
As estratégias de redução dos desastres ambientais ${ }^{10}$ sejam eles naturais ou provocados pela ação humana, e o gerenciamento dos riscos de desastres são modos de lidarem com os mesmos e que buscam reduzir a vulnerabilidade e oferecer capacidade para suportar adaptações, fazendo com que essas pessoas mais vulneráveis consigam lidar com desastres naturais como secas, tempestades assim como os riscos ambientais provocados por fatores humanos.

Nesse sentido, o documento internacional para redução dos riscos de desastres, realizada no Japão em 2005 denominado de "Hyogo Framework for Action 2005-2015 Building the Resilience of Nations and Communities to Disasters ${ }^{\prime 11}$, considera a relação entre risco ambiental, desenvolvimento sustentável, desenvolvimento de mecanismos e capacidades na construção de barreiras contra os perigos e a erradicação da pobreza. Dessa forma, a Declaração de Kobe é um importante instrumento para a redução da vulnerabilidade para os riscos de desastres ambientais.

Ainda, a Declaração de Hyogo afirma que os riscos de desastres ambientais aumentam quando os perigos interagem com vulnerabilidades físicas, sociais econômicas e ambientais. Apesar do crescente entendimento e aceitação da importância da redução do risco de desastres ambientais e o aumento da capacidade de responder a eles; os desastres e principalmente a gestão e redução dos riscos continuam a ser um grande desafio global.

Portanto, os riscos de desastres surgem quando o perigo interage com vulnerabilidades físicas, sociais, econômicas e ambientais, ou seja, se as vulnerabilidades fossem minimizadas as situações de perigo ambiental não se tornariam desastrosas. O perigo é intensificado pela vulnerabilidade, a qual contribui consideravelmente para a concretização dos riscos. ${ }^{12} \mathrm{O}$ conceito de vulnerabilidade está ligado às relações que as pessoas tem com o seu meio ambiente e com a força social e valores culturais que sustentam essa interligação.

A ocorrência de desastres ambientais e o risco de desastres, derivado deste são causas principais e importantes no fluxo migratório das pessoas, mais migrações acontecem devido a gradual degradação do meio ambiente. A maioria das migrações ambientais acontecem entre países, mas se torna relevante a evidencia que a degradação ambiental está influenciando também migrações internacionais. ${ }^{13} \mathrm{~A}$ especial vulnerabilidade dos migrantes se origina no fato de que eles não são cidadãos do país aos quais se encontram, eles ultrapassaram a fronteira internacional e eles têm muitas vezes que entrar e viver em outro país apenas com a autorização das autoridades; e com isso essa vulnerabilidade contrasta com o poder de recuperação que exige o processo de migração.

Diante disso, percebe-se o drama das migrações ambientais que acontecem em resposta aos desastres ambientais ou ao medo do risco de desastre ou calamidade. Enquanto a causa de tais migrações seria a degradação do meio ambiente é muito importante a dimensão social de tais movimentos migratórios. Hugo Graeme afirma que "os países e grupos mais pobres estão em desvantagem, pois eles não têm os recursos para assimilar e praticar sofisticados sistemas de alerta ou para fundar um rápido e bem planejado modo de prevenção e precaução de desastres e conseqüentemente assistir as vitimas em sua recomposição. ${ }^{14}$ 
Por isso, se torna aparente que alguns locais ou regiões, especialmente os que se encontram em países menos desenvolvidos, são particularmente vulneráveis a degradação ambiental e conseqüentemente são os que mais produzem migrantes ambientais, que migram dentro de seus países e também fora de suas fronteiras.

Neste sentido ressalta Hugo Graeme examinando os impactos da degradação ambiental na população "que é importante lembrar que a migração é a única forma que essas populações afetadas encontram como resposta e deve ser discutido que há necessidades mais importantes, em particular a adaptação dessas pessoas aos efeitos dos processos ambientais. ${ }^{15}$

Os riscos de desastres e os impactos adversos dos perigos naturais podem ser reduzidos pelo monitoramento, análise e gerenciamento das causas de desastres, incluindo a redução das vulnerabilidades sociais e econômicas e dando suporte para que essas pessoas respondam o mais rápido possível as adversidades dos eventos ambientais perigosos.

Os maiores elementos que dão magnitude ao risco ambiental são os perigos, ou seja, o potencial de eventos danosos, e a vulnerabilidade da população a esses perigos, pois os desastres ambientais são a combinação de uma população ou comunidade vulnerável com um evento perigoso que resulta em um desastre.

Tanto desastres naturais quanto desastres que ocorrem pela interferência humana, ou acidentes industriais estão ganhando cada vez mais repercussão, principalmente no que diz respeito as conseqüências humanas. O Programa de Desenvolvimento das Nações Unidas indica a forte inter relação entre o nível de desenvolvimento de um país e o nível de mortalidade diante desses desastres. Há muitas regiões no mundo as quais são extremamente vulneráveis aos desastres ambientais, de origem natural ou humana. A realidade desses recentes desastres $e$ o crescimento dos riscos ambientais não são adequadamente considerados nos instrumentos jurídicos internacionais ou domésticos. ${ }^{16}$

Com isso, percebe-se que os desastres ambientais e seus riscos não atingem a todos indistintamente, no que diz respeito a vulnerabilidade. Entre os fatores que podem gerar uma maior vulnerabilidade para a prevenção e enfrentamento dos riscos de desastres ambientais se destaca a pobreza, pois afeta consideravelmente a capacidade de determinados indivíduos ou comunidade de prevenir e se proteger dos desastres e riscos ambientais.

Sabe-se que as medidas a serem adotadas para a redução do risco de desastres e de suas conseqüências passam necessariamente pelo enfrentamento da vulnerabilidade gerada pela pobreza, e as desigualdades na distribuição dos riscos e custos ambientais decorrentes. Devido a isso, é que se coloca a íntima relação entre vulnerabilidade ambiental e violação de direitos humanos, pois os mais pobres são os mais atingidos pela distribuição dos custos ambientais bem como pelos riscos, e os mais afetados em sua dignidade humana.

A obediência as sugestões de esforços para a redução da vulnerabilidade e a construção do poder de recuperação a eventos extremos devem ser tidos como 
prioridade a longo e curto prazo. Essa priorização ajudaria prevenir as perdas humanitárias e econômicas a curto prazo, assim como ganhos no desenvolvimento e a promoção de uma base sustentável para outras ações de adaptação a longo prazo. Com isso, se capitalizaria um grande conhecimento e capacidades, especialmente na redução de risco de desastres e no gerenciamento dos riscos.

Em virtude dessas questões, as pessoas mais vulneráveis aos riscos de desastres ambientais são afetadas consideravelmente no que diz respeito aos direitos humanos reconhecidos de forma internacional, os quais em situações de riscos ambientais e desastres ecológicos se veem violados, como o direito a vida, a saúde, a dignidade humana entre outros.

As condições ambientais desfavoráveis podem ser causa ou conseqüência da violação dos direitos humanos, principalmente porque as pessoas ou grupos mais pobres e que se encontram em uma posição desprivilegiada para exercer estes direitos são as vítimas principais e preferenciais dos riscos e custos ambientais. ${ }^{17}$

O processo de violação dos direitos humanos alcança principalmente os grupos sociais vulneráveis, neste contexto, os grupos de populações mais pobres. De acordo com Piovesan "a efetiva proteção dos direitos humanos demanda não apenas políticas universalistas, mas específicas, endereçadas a grupos socialmente vulneráveis, enquanto vítimas preferenciais da exclusão."18

Diante disso, e principalmente da questão ambiental e suas implicações sociais, o Relatório de Desenvolvimento Humano 20072008 do Programa das Nações Unidas para o Desenvolvimento através da resolução 723 denominada Mudanças Climáticas e Direitos Humanos, revela para um quadro preocupante e injusto no horizonte humano, com um mundo cada vez mais dividido entre nações ricas altamente poluidoras e países pobres. O mais importante nesta resolução é o reconhecimento da vulnerabilidade dos mais pobres, especialmente os que vivem em zonas de alto risco frente aos riscos ambientais e desastres ecológicos e sua capacidade de adaptação mais limitada, o que os torna também mais vulneráveis as violações dos direitos humanos originadas em razão dos riscos e dos desastres ecológicos.

Por isso, as pessoas mais vulneráveis aos efeitos dos riscos e desastres ambientais provocados de forma natural ou pela ação do homem serão aquelas mais pobres, as quais já possuem uma condição de vida precária, desprovidas do acesso aos seus direitos humanos básicos como moradia adequada, saúde, saneamento básico, educação, alimentação, dignidade, entre outros.

Embora haja potencialmente riscos de catástrofes ambientais para todos, a distribuição dos custos e benefícios a curto e médio prazo estará longe de ser uniforme. Os riscos ambientais e sociais são distribuídos de forma desigual, ocasionando uma injustiça ambiental, pois os países mais responsáveis e que contribuem de forma direta com a degradação ambiental serão os menos afetados, enquanto os países em desenvolvimento, que estão sujeitos aos mesmos riscos ambientais, serão os mais afetados, em virtude da relação entre pobreza e vulnerabilidade. 


\title{
2 PROTEÇÃO E RESPONSABILIDADE DO ESTADO DIANTE DOS MIGRANTES AMBIENTAIS
}

Uma clareza e coerência nos instrumentos internacionais de proteção aos direitos humanos das pessoas deslocadas ou que necessitam migrar em razão do risco e de desastres ambientais são essenciais, mesmo que ainda não exista nenhum documento ou convenção relativa especificamente a esses migrantes ambientais. O que se torna indispensável é a efetiva atuação dos Estados-nações no que diz respeito a proteção do meio ambiente, dos direitos humanos fundamentais das pessoas atingidas pelo risco e desastres ambientais, adotando medidas de prevenção e precaução.

As pessoas deslocadas por desastres ambientais ou riscos ambientais, sejam eles naturais ou muitas vezes provocadas pela ação do homem e sua negligência, tais como: desastres nucleares, acidentes internacionais de poluição da água, mudanças climáticas e o aquecimento global entre outras, constituem uma categoria que requer proteção. Dessa forma, percebe-se o vínculo entre os fluxos de migrantes por razões ambientais e a proteção de direitos humanos, pois se está diante de violações graves de direitos humanos.

Em busca da proteção dos direitos humanos dos migrantes ambientais afirma Trindade:

\begin{abstract}
A proteção de refugiados e pessoas deslocadas há assim de ser apropriadamente coordenada com os mecanismos de proteção dos direitos humanos (a níveis global e regional) $\mathrm{E}$ a emergência de coletividades humanas em necessidade de atenção e proteção especiais - pessoas internacionalmente deslocadas, pessoas internamente deslocadas, os repatriados, os assim-chamados "migrantes econômicos", etc - dá uma dimensão nova e mais ampla ao direito internacional dos refugiados. ${ }^{19}$
\end{abstract}

No caso dos migrantes ambientais, a proteção dos direitos humanos destes grupos para Piovesan "torna-se, contudo, insuficiente tratar o indivíduo de forma genérica, geral e abstrata. Faz-se necessária a especificação do sujeito de direito, que passa a ser visto em sua peculiaridade e particularidade" ${ }^{\prime 20}$ Dessa forma, a violação de direitos humanos de alguns grupos, principalmente os vulneráveis aos riscos e desastres ambientais, bem como os que já se encontram em processo de migração, exigem uma resposta específica e diferenciada.

Em primeiro lugar é responsabilidade do Estado em providenciar aos seus cidadãos os direitos humanos básicos diante do impacto e das conseqüências dos riscos e desastres ambientais. Se a responsabilidade do Estado não é suficiente, é que há a responsabilidade da comunidade internacional em intervir na situação para garantir os direitos humanos básicos das pessoas.

Por isso, aos riscos e desastres ambientais se aplicam, além do dever geral de toda sociedade global de preveni-los, também o dever geral de minimizar os danos e prover assistência humanitária de emergência, incluindo as obrigações de notificar de imediato e de fornecer informações, de desenvolver planos para as contingências, e de cooperação entre os Estados para minimizar os danos e 
compensar pelos danos deles resultantes. ${ }^{21} \mathrm{Na}$ mesma perspectiva de pensamento Nurith Kliot assinala que um grande problema a ser resolvido quanto a migração ambiental é sobre as perspectivas quanto aos direitos. Direitos representam a crença que todas as pessoas merecem respeito e uma solução para seus problemas, que seria a criação de condições necessárias para prover significativa ajuda para os países de onde vêem esses migrantes. ${ }^{22}$

O problema dos migrantes ambientais promete estar no topo da maior crise humana em nossos tempos e assim que esta problemática se torna mais opressiva, as responsabilidades da sociedade e dos Estados se tornam desafios cada vez mais preocupantes. $^{23}$

Com isso, a proteção aos direitos humanos dos migrantes ambientais é um assunto que deve ser uma das maiores preocupações da comunidade internacional, pois como afirma Kliot "o meio ambiente saudável é apenas uma das condições necessárias para o ser humano, mas o único que fornece o fundamento para muitos outros direitos". ${ }^{24}$

Nesta mesma perspectiva, Laura Westra assinala a responsabilidade dos Estados na proteção dos seres humanos, indo além da perspectiva de seus cidadãos quando os migrantes ambientais atravessam as fronteiras de seus países:

\begin{abstract}
Estados devem a proteção física para todos os seus cidadãos, de acordo com os instrumentos domésticos, como as Constituições. Mas o respeito a todos os seres humanos é uma obrigação internacional "erga onmes" e nenhum estado individualmente pode impor condições e clamar legalidade quando migrantes aparecem em seus limites fronteiriços. [...] No entanto, respeito pela dignidade humana é pouco provido quando o auxílio ao migrante é dado. ${ }^{25}$
\end{abstract}

O grande desafio da comunidade internacional com relação às políticas púbicas de segurança humanitária e a proteção aos direitos humanos são de grande importância, e os migrantes ambientais são reconhecidos hoje como uma categoria separada, os quais não há muito conhecimento nas leis, mas estão cada vez aumentando por causa da degradação ambiental a longo e curto prazo, o que agrava a situação criada pelos impactos dos desastres.

O fenômeno do deslocamento de indivíduos e comunidades em razão de risco e desastre ambiental traz um grande desafio ao direito ambiental e aos sistemas de proteção aos direitos humanos. Por isso, se faz necessária uma resposta efetiva no plano jurídico internacional a situação dos migrantes ambientais.

É importante salientar que nos dias de hoje, no Estado atual do direito internacional, há a inexistência de normas jurídicas destinadas a proteção dos deslocados ou migrantes ambientais, principalmente no que diz respeito aos movimentos interestatais, ou seja, os que ocorrem de forma internacional ultrapassando os limites dos países. ${ }^{26}$

Uma possibilidade seria a formatada por Véronique Magniny ${ }^{27}$, a qual prevê a criação de uma nova convenção, que se baseasse em princípios de direito e que 
tivesse na proteção dos direitos humanos seu maior fundamento, atribuindo responsabilidades diferenciadas aos seus signatários de acordo com o grau de contribuição de cada um para as migrações. Ainda acrescenta um estatuto coletivo dos deslocados ambientais, que não distinguisse as causas da partida ou dos desastres, mas que agisse sobre suas conseqüências seria muito mais realista.

Percebe-se que o aprimoramento da legislação, quer seja através da elaboração de acordos bilaterais, de abrangência regional, ou pela formulação de uma convenção universal, que reconheça tal categoria, seria importante na resolução do problema dos deslocados ambientais.

Segundo Christel Cournil, todas as alternativas de proteção aos migrantes ambientais de seguir alguns pressupostos, entre eles, a exigência de uma base científica consolidada sobre a problemática dos migrantes e sua relação com o meio ambiente, que possa embasar, através da demonstração das causas e da projeção de cenários, as decisões e a formulação de políticas de amparo as pessoas afetadas; o aumento da conscientização da sociedade sobre a importância dos debates destes temas, atentado-se para as mudanças que ocorrerão nos meios sociais, econômicos e culturais a partir da tentativa de inserção dos grupos deslocados; o oferecimento dos meios adequados para a ajuda humanitária, por meio da capacitação dos recursos humanos das entidades que auxiliam as Nações Unidas e do apoio material e logístico necessários para a mitigação dos resultados e adaptação das pessoas atingidas e, por fim, o autor sugere que conceitos sejam concebidos,, no sentido de fortalecer as políticas públicas bem como sejam fortalecidas e criadas, quando necessárias, instituições públicas e privadas para tratarem da questão do fluxo de migrantes ambientais, tanto a nível nacional quanto internacional, mesmo que, para isso, se arquitete um novo órgão, vinculado as Nações Unidas, para cuidar, especialmente, do estudo e da elaboração de estratégias para estes grupos. ${ }^{28}$

A partir dessas alternativas e anseios quanto a proteção desses indivíduos e populações que tem que se deslocar em função de riscos e desastres ambientais, não apenas no interior de um mesmo país mas também entre estados, de forma internacional, é que pode-se constatar que os deslocamentos internos foram objeto de especial atenção por parte dos organismos internacionais.

Entretanto, os deslocados internos ainda não contam com um instrumento jurídico específico, mas o Escritório do Alto Comissariado das Nações Unidas para os Direitos Humanos lançou um conjunto de Princípios Orientadores Relativos aos Deslocados Internos. 29

Mas em função dos deslocamentos ambientais, não só internos como interestatais merece destaque o "Projeto de Convenção sobre o Estatuto Internacional dos Deslocados"30 ambientais, publicado na Revista Europeia de Direito Ambiental (Revue Européenne de Droit de l'Environment) realizado por um grupo de trabalho da Universidade de Limoges liderados pelo professor Michel Prieur. Este grupo de trabalho foi composto pelo CRIDEAU (Centre de recherches interdisciplinaires em droit de l'environnement, de l'aménagement et de l'urbanisme) e do CRDP (Centre de recherches sur lês droit de La personne, equipes temáticas 
do OMIJ (Observatoire dês mutations institutionnelles et juridiques), com o apoio do CIDCE (Centre International de Droit Comparé de l'Environnement). ${ }^{31}$

Esse projeto de convenção utiliza-se da expressão "deslocados ambientais", ao invés de "migrantes", mas propõe uma classificação muito semelhante àquela feita pela IOM (2008), sendo possível uma leitura similar dos termos. De acordo com tal documento, são conceituados de "deslocados ambientais" como:

[...] as pessoas físicas, as famílias e as populações confrontadas a um desastre brutal ou gradual em seu ambiente afetando inelutavelmente suas condições de vida e Ihes forçando a deixar, com urgência ou no seu decorrer, seus lugares habituais de vida e requerendo sua relocação ou realojamento. ${ }^{32}$

O projeto optou pela expressão "deslocados", pois é majoritariamente utilizada em textos oficiais, além de melhor refletir a diversidade de causas e modalidades dos deslocamentos e o seu caráter coletivo, além de dar a clareza de que é um deslocamento forçado e não espontâneo.

Este documento faz referencia aos desastres ambientais repentinos e aqueles que gradualmente, vão alterando o meio ambiente, ou seja, o risco ou ameaça ambiental da ocorrência dos desastres, os quais forçam as pessoas a deslocaremse. Conforme os autores do projeto, os eventos ambientais podem ter sua origem em causas naturais ou pela ação humana e sua influencia na degradação dos meios de sobrevivência.

Ainda, o artigo $3^{\circ}$ de tal projeto verifica o seu âmbito de aplicação que é universal, não havendo distinção entre pessoas que se deslocam entre um e outro país e pessoas que se deslocam internamente, dentro de seus próprios Estados. ${ }^{33}$ Esta é a grande mudança neste projeto de convenção abrangendo as pessoas que se deslocam além das fronteiras de seus países de forma internacional e não apenas aquelas que migram dentro dos limites de seus países.

A proposta da Convenção tem como grande objetivo contribuir para garantir direitos aos deslocados ambientais e organizar seu acolhimento e eventual retorno aos seus lugares, regiões de origem. Com vistas à garantia desses direitos humanos o projeto elenca os princípios da responsabilidade comum, mas diferenciada, da proximidade, da proporcionalidade, da efetividade e da não-discriminação.

Nesse ponto esclarecem Cavedon e Vieira que o projeto reconhece como direitos comuns aos deslocados ambientais o direito a informação e participação; direito a assistência e socorro; direito a água e a ajuda alimentar; direito a habitação; direito ao cuidado médico; proteção dos direitos da pessoa, entre diversos outros direitos humanos básicos para sobrevivência. Ainda, as famílias e populações deslocadas têm o direito a preservação da unidade familiar e as populações são beneficiadas pelo regime jurídico as minorias, nos países de acolhida. ${ }^{34}$

As migrações ambientais geradas muitas vezes, por ameaças/riscos e desastres ambientais sejam eles naturais ou pela ação humana, além de ser uma questão ambiental é também uma questão relevante de direitos humanos, pois a 
vulnerabilidade ambiental a esses fatores ambientais são capazes de gerar extremas violações aos direitos humanos dos migrantes.

Diante da análise de tal documento, entende-se que se o mesmo for efetivamente adotado, pode ser o grande pioneiro na articulação entre o direito ambiental e os sistemas de proteção dos direitos humanos, pois são notórias as conexões entre as vulnerabilidades ambientais com a realização da justiça ambiental e o estabelecimento de formas eficazes de prevenção, precaução e gestão dos riscos e desastres ambientais.

Ainda, os sistemas regional e internacional de proteção aos direitos humanos podem se constituir de destacados espaços na proteção dos migrantes ambientais ante aos riscos e desastres ambientais, incorporando aos mesmos as normas ambientais e os princípios jurídicos do direito ambiental, principalmente o princípio da precaução, na análise das violações dos direitos humanos.

\section{RISCOS E DESASTRES AMBIENTAIS E A SUA GESTÃO NA MITIGAÇÃO DE MIGRAÇÕES LOCAIS E INTERNACIONAIS}

Além da preocupação com a proteção e a responsabilidade dos Estados diante dos das migrações em nível local e internacional, é também importante ressaltar as tentativas da comunidade internacional em reduzir os riscos de desastres ambientais. Esses esforços devem ser relacionados com as políticas, planos e programas para o desenvolvimento sustentável e a redução da pobreza, baseadas na cooperação internacional e regional.

Entretanto, o grande problema não é proteger e dar assistência as pessoas forçadas a migrarem devido às causas ambientais associados a outros fatores, mas sim adotar políticas as quais irão lidar com essas causas e tentar fazer com que as mesmas se tornem desnecessárias ${ }^{35}$.

Os riscos e desastres ambientais e seus impactos adversos podem ser reduzidos mediante monitoramento, análise sistemática, gerenciamento e precaução das causas dos desastres, incluindo a redução social e econômica das populações vulneráveis ambientalmente, bem como melhorando o preparo dos indivíduos e das comunidades em responder as adversidades dos eventos ambientais perigosos.

O documento internacional preparado pela IASC e pela ISDR (International Strategy for Disaster Reduction) ${ }^{36}$ titulado de Disaster Risk Reduction Strategies and Risk Management Practices: Critical Elements for Adaptation to Climate Change ${ }^{37}$ de 11 de novembro de 2008 traz que os dois principais elementos que dão magnitude aos riscos são os perigos, ou seja, os potenciais eventos catastróficos ou fenômenos naturais, e a vulnerabilidade da população a esses perigos. Ainda, acrescenta que as atividades humanas podem aumentar o nível do risco de desastres ambientais.

Baseado nos conceitos deste documento, a Declaração de Hyogo coloca as estratégias para a redução dos riscos de desastres através de cinco prioridades para ação: 
1. Assegurar que a redução do risco de desastre é uma prioridade nacional e local com forte base institucional para implementação.

2. Identificar, estimar e monitorar o risco de desastre e intensificar os sistemas de alarme preventivos.

3. Usar do conhecimento, inovação e educação para construir uma cultura de segurança e resiliência em todos os níveis

4. Reduzir os fatores de riscos

5. Fortalecimento para efetiva resposta de prontidão aos desastres. ${ }^{37}$

Portanto, para a redução do risco de desastres ambientais os Estados, de forma local e internacional, bem como organizações preocupadas com essa questão devem levar em consideração as prioridades citadas acima e devem programá-las com suas próprias circunstancias e capacidades.

Entretanto, há também a preocupação com a gestão dos riscos ambientais pelos países, que deve lidar com a incerteza e o potencial das perdas, envolvendo dessa forma, a análise e o desenvolvimento de estratégias e específicas ações para controlar e reduzir a produção de riscos.

A gestão dos riscos é um termo usado para a prática do controle da incerteza e as potencias perdas envolvendo a avaliação dos riscos, análise, desenvolvimento de estratégias e específicas ação para controlar e reduzir os riscos e perdas. A redução dos riscos e dos desastres podem se dirigir as medidas de prevenção, sistemas de alerta imediato e resposta a extensão dos eventos ambientais extremos.

Com a execução de práticas que levam a redução dos riscos e dos desastres ambientais e a gestão dos riscos o que se tem por conseqüência é a mitigação das migrações em função destes aspectos, seja de forma local ou internacional. Contudo, essas práticas não irão eliminar por completo a necessidade de uma resposta humanitária aos eventos extremos da natureza, particularmente nas comunidades mais vulneráveis.

Devido a isso, se faz necessário levar a prática de políticas de prevenção, com o propósito de mitigar a necessidade de migrar, assegurando a essas pessoas um meio ambiente de vida aceitável estabelecendo uma moradia digna. ${ }^{38}$

Diante deste aspecto, Hermsmeyer acrescenta que está estabelecido dessa forma que os migrantes ambientais tem sido e continuarão a ser um incontestável resultado da severa degradação ambiental, alem disso, eles necessitam de uma garantia suficiente para seus direitos humanos básicos, quando os Estados nacionais não podem ou não protegem os mesmos. ${ }^{39}$

Pode-se colocar em evidencia que o principio da precaução deve servir como base das políticas para aproximar os discursos da relação entre degradação ambiental e a migração. Com isso, faz-se necessário apontar algumas sugestões de políticas para mitigação dos riscos, desastres ambientais e assim reduzir o número de pessoas que necessitam migrar que devem ser implementadas em paralelo aos esforços com os esforços para redução da pobreza. 
As alternativas de políticas apontadas começam com a exigência de uma forte base científica, pois há a necessidade de colocar em prática programas para permitir um melhor entendimento das causas e efeitos entre degradação ambiental e as migrações das populações. É importante também elevar a atenção pública no assunto das migrações ambientais e nas dimensões sociais, econômicas e ambientais decorrentes delas. Essa etapa é importante assim como o debate sobre as migrações na agenda de muitos países e específicas regiões, e é necessário que os migrantes sejam comunicados de forma mais eficiente em todo o mundo.

Nessa perspectiva, Myers acrescenta a necessidade de adotar políticas as quais irão lidar com as causas das migrações ambientais, e consequentemente torná-las desnecessárias, o qual inclui a promoção do desenvolvimento sustentável; capacidade de ajuda estrangeira designada para aliviar as pressões ambientais e tratar as necessidades dos grupos mais pobres; capacidade de diminuir o débito das nações mais pobres e iniciativas específicas preparadas para ajudar países desenvolvidos para confrontar os desafios ambientais. ${ }^{40}$

Ainda, Myers aponta que é preciso um entendimento profundo e sábio a respeito das migrações ambientais estabelecendo as causas do problema, mas não apenas causas ambientais, mas problemas associados como a segurança, a vulnerabilidade das populações bem como a problemática econômica de alguns países. $^{41}$

A promoção de uma cultura de prevenção e precaução, incluindo a mobilização de recursos adequados para a redução do risco e dos desastres ambientais é um investimento para o futuro com retorno substancial. A gestão do risco e os sistemas de alerta imediato são essenciais investimento para proteger e salvar vidas e meios de vida, e ainda contribuem para o desenvolvimento sustentável e são mais efetivos.

Assim que a situação das migrações ambientais for reconhecida internacionalmente, esses indivíduos devem ter garantidos seus direitos humanos básicos e a magnitude desse problema pode ser reduzido substancialmente. À medida que as causas das degradações ambientais podem ser efetivamente mitigadas as pressões que forçam as populações a migrarem de seus ambientes podem ser também reduzidas.

Diante disso, todos os problemas ambientais geradores dos fluxos migratórios podem ser reduzidos através de uma cooperação e motivação internacional, isso limitaria o tamanho e a enormidade do problema e a pressão que seria colocada na economia, política e estabilidade social do mundo.

\section{CONCLUSÃO}

Independentemente do instrumento jurídico que for utilizado para proteger os deslocados ambientais, este deverá prever mecanismos de amparo aos grupos que movimentam-se dentro dos seus próprios Estados, desenvolvendo-se formas de acesso aos territórios através dos princípios de cooperação internacional, hospitalidade universal e obrigação de assistência humanitária, em caso de catástrofes ambientais, bem como do princípio da solidariedade internacional que 
pressupõe que as nações tem um dever mútuo de prestarem auxílio quando da ocorrência de eventos naturais insuperáveis. Não se deve deixar de determinar responsabilidades aos países que mais contribuíram para as causas que originaram as migrações, fundamentando-se esta conclusão no princípio proclamado pela Convenção do Rio da responsabilidade comum, mas diferenciada.

Os Estados, possuem obrigações comuns de auxiliar aos povos afetados pelos efeitos das alterações climáticas, exigindo-se, no entanto, daqueles que, através de suas ações, tiveram um grau maior de participação na origem dos eventos que resultaram em deslocados, uma imputação diferenciada de atribuições que permitam aos países atingidos minimizarem as conseqüências das alterações em seu ambiente, mitigarem os efeitos econômicos e sociais à população e possibilitarem que se garanta um reassentamento seguro e eficiente nos casos em que não é possível o retorno, quando, por exemplo, ocorrerem inundações pela elevação do nível do mar.

Além de ampliar a prevalência das estratégias de prevenção e precaução, o reconhecimento internacional dos migrantes ambientais deveria também estimular 0 desenvolvimento de uma compreensiva e efetiva estrutura de responsabilidade e ação internacional. Diante dos desastres e riscos ambientais, pode-se constatar que os mesmos podem ser mitigados se as autoridades, indivíduos e comunidades em zonas de perigo estiverem bem preparados e prontos para agir e equipados com conhecimento e capacidades para uma efetiva gestão do risco e dos desastres.

\title{
MIGRATION, VULNERABILITY E (IN) AMBIENT JUSTICE: CHALLENGES AND PERPECTIVAS
}

\begin{abstract}
The environmental degradation reaches alarming levels and the ecosystems destruction come provoking the migration forced of people reached by environmental catastrophes around the world. The forms and the consequences of these aggressions to the environment took over, in the contemporaneousness, disturbing levels, mostly by the performance of the industrialized countries and of the society, great producers of ecological risks, turning vulnerable people and carrying what can call environmental (in)justice.
\end{abstract}

Keywords: Degradation. Injustice. Migration. Vulnerability.

\section{NOTAS}

Doutora em Direito - Teoria e Filosofia do Direito pela Universidade Federal do Paraná. PósDoutora em Direito Ambiental e Antropologia Jurídica pela Universidade Federal de Santa Catarina. Professora do Programa de Mestrado em Direito Ambiental da UCS e do Mestrado em Política Social da UCPEL. E-mail: rsberguer@main.unijui.tche.br. Endereço: Universidade Católica de Pelotas, Centro de Ciências Jurídicas Sociais e da Administração - Rua Félix da Cunha, 412 - Centro - CEP 96010-000 - Pelotas, RS - Brasil. 
2 Mestre em Direito Ambiental pela UCS. Advogada. E-mail: v_vergani@brturbo.com.br. Endereço: Vergani Advogados Associados - Avenida Júlio de Castilhos, 1188 sala 32 - Caxias do Sul, RS, Brasil.

3 TRINDADE, Antônio Augusto Cançado. Direitos Humanos e meio ambiente: paralelo dos sistemas de proteção internacional. Porto Alegre: Fabris, 1993. p.89.

4 BECK, Ulrich. La sociedad del riesgo hacia una nueva modernidad. Barcelona Paidós, 1998.

5 BECK, Ulrich. La sociedad del riesgo hacia una nueva modernidad. Barcelona Paidós, 1998, p. 40.

6 CAVEDON, F. S.; VIEIRA, R. S. Socioambientalismo e Justiça Ambiental. In. BENJAMIN, A. H. ; LECEY, E. ; CAPPELLI, S. (Org.). Mudanças Climáticas, Biodiversidade e Uso Sustentável de Energia. São Paulo : Imprensa Oficial do Estado de São Paulo, 2009.p.546.

7 WESTRA, Laura. Environmental justice and the rights of ecological refugees. London: Earthscan. Editora, 2009. Traduzido pelas autoras [...] environmental disasters are traditionally classified into three categories 1 long-term environmental degradation, including global warming, deforestation, land erosion, salinity, waterlogging and desertigication; 2 sudden natural environmental disruptions, including earthquakes, droughts, floods, hurricanes, monsoons, tidal waves, tornadoes and volcanic eruptions; 3 accidents, including both industrial and chemical disasters.

8 IASC é um fórum inter-agencias único, criado em 1992 pela Assembléia Geral das Nações Unidas que tem como funções as de coordenação, desenvolvimento de políticas e processos decisórios envolvendo parceiros humanitários tanto do sistema das Nações Unidas como externos. (2008, versão piloto).

9 Traduzido pelas autoras: Natural disasters are the consequences of events triggered by natural hazards that overwhelm local response capacity and seriously affect the social and economic development of a region. Traditionally, natural disasters have been seen as situations that create challenges and problems mainly of a humanitarian nature. However, increasingly, it has come to be recognized, that human rights protection also needs to be provided in these contexts.

10 Trindade (1993), nessa senda, propõe a distinção entre desastres naturais e desastres ambientais, atribuindo àqueles os eventos naturais "puros", que ocorrem sem a interferência humana, tais como vulcões, relâmpagos, terremotos, furacões e, a estes, os fenômenos aos quais os homens têm responsabilidade, direta ou indiretamente, como vazamentos de óleo, incêndios florestais e, inclusive, eventos causados pelas mudanças climáticas, como secas, inundações e aumento do nível do mar.

11 Declaração resultante da Conferência Mundial para Redução dos Desastres, realizada em Kobe no Japão em Janeiro de 2005 por 168 países e aprovada pela Assembleia Geral das Nações Unidas. A Conferencia Mundial sobre Redução de Desastres 2005-2015 construindo a resiliencia de nações e comunidades para os desastres. Disponível em: http://www.unisdr.org/eng/hfa/hfa.htm. Acesso em 19 de fevereiro de 2010.

12 CAVEDON, F. S.; VIEIRA, R. S. Socioambientalismo e Justiça Ambiental. In. BENJAMIN, A. H. ; LECEY, E. ; CAPPELLI, S. (Org.). Mudanças Climáticas, Biodiversidade e Uso Sustentável de Energia. São Paulo : Imprensa Oficial do Estado de São Paulo, 2009.p.547.

13 HUGO, Graeme. Migration, development and environment. IOM Imigration Research Series. n. 35. Genebra: IOM, 2008.

14 Traduzido pelas autoras. Poorer countries and groups can be at a disadvantage because they do not have the resources to put in place sophisticated warning systems or to fund a rapid, planned, well provisioned flight from the disaster and to subsequently assist the victims to recover. Cfe. HUGO, Graeme. Migration, development and environment. IOM Imigration Research Series. n. 35. Genebra: IOM, 2008. p.14.

15 Traduzido pelas autoras. In the examination of the impact of environmental degradation on population it is important to remember that migration is only one of the ways in which populations effected respond, it can be argued that there needs to be more attention paid to other responses, in particular adaptations to the effects of environmental processes. Cfe. HUGO, Graeme. Migration, development and environment. IOM Imigration Research Series. n. 35. Genebra: IOM, 2008. p.14.

16 WESTRA, Laura. Environmental justice and the rights of ecological refugees. London: Earthscan., 2009 , p. 80.

17 CAVEDON, F. S.; VIEIRA, R. S. Socioambientalismo e Justiça Ambiental. In. BENJAMIN, A. H. ; LECEY, E. ; CAPPELLI, S. (Org.). Mudanças Climáticas, Biodiversidade e Uso Sustentável de Energia. São Paulo : Imprensa Oficial do Estado de São Paulo, 2009.p.550.

18 PIOVESAN, Flávia. Direitos humanos e o direito constitucional internacional. São Paulo: Max Limonad, 2007.p.27-8. 
19 TRINDADE, Antônio Augusto Cançado. Direitos Humanos e meio ambiente: paralelo dos sistemas de proteção internacional. Porto Alegre: Fabris, 1993. p.137.

20 PIOVESAN, Flávia. Direitos humanos e o direito constitucional internacional. São Paulo: Max Limonad, 2007.p.28.

21 BROWN, Oli. Migration and climate change. IOM Imigration Research Series, n. 31. Genebra: IOM, 2008.p.141-169.

22 KLIOT, Nurit. Environmentally induced population movements their complex sources and consequences in KLIOT, Nurit; KROL, Maarten e UNRUH, Jon. Environmental change and it implications for population migration. Norwell, Kluwer Academic Publishers, 2004. p.77. MYERS, Norman. Environmental Refugees: An emergent security issue. Prague: XVIII Economic Forum. 23-27 mai 2005. Disponível em: <http://www.osce.org/ documents/eea/2005/05/14488 en.pdf> Acesso em: 30 mar 2009.

$24 \mathrm{KLIOT}$, Nurit. Environmentally induced population movements their complex sources and consequences in KLIOT, Nurit; KROL, Maarten e UNRUH, Jon. Environmental change and it implications for population migration. Norwell, Kluwer Academic Publishers, 2004. p.71.

Traduzido pelas autoras: States owe physical protection to al their citizens, according to domestic instruments, such as constitutions. But respect for all human beings is an international obligation erga onmes and no individual state can impose inhumane conditions and claim legalitu for its actions when asylum seekers show at their borders. [...] However, respect for human dignity is normally in short supply when refugee aid is provided. WESTRA, Laura. Environmental justice and the rights of ecological refugees. London: Earthscan, 2009.

CAVEDON, F. S.; VIEIRA, R. S. Socioambientalismo e Justiça Ambiental. In. BENJAMIN, A. H. ; LECEY, E. ; CAPPELLI, S. (Org.). Mudanças Climáticas, Biodiversidade e Uso Sustentável de Energia. São Paulo : Imprensa Oficial do Estado de São Paulo, 2009.p.557.

27 MAGNINY. Véronique. Des victimes de l'environnement aux réfugiés de l'environnement. In: Revue Asylon(s), n. 6, nov 2008. Disponível em: <http://terra.rezo.net/article845.html>. Acesso em: 18 março 2010.

COURNIL, Christel. À la recherche d'une protection pour les 'réfugiés environnementaux': actions, obstacles, enjeux et protections. In: Revue Asylon(s), n. 6, nov 2008. Disponível em:<http://terra.rezo.net/article843.html>. Acesso em: 19 mar 2010.

29 Traduzido pelas autoras: Guiding Principles on Internal Displacement.

30 Traduzido pelas autoras: Projet de convencion relative au statut international des déplacésenvrironnementaux.

31 PRIEUR, Michel et al. Projet de convention relative au statut international des déplacés environnementaux. In: Revue européenne de droit de l'environnement. n. 4, 2008. Disponível em: <http://www.cidce.org/pdf/Projet\%20de\%20convention\%20relative\%20au\% 20statut\%20international\%20des\%20déplacés\%20environnementaux.pdf>. Acesso em: 20 fev. 2010.

32 Traduzido pelas autoras: [...] personnes physiques, les familles et les populations confrontées a um bouleversement brutal ou insidieux de leur environment portant inéluctablement atteite a leurs conditions de vie et lês forçant a quitter, dans l'urgence ou dans La durée, leurs lieux habituels de vie et conduisant a leur réinstallation et a leur relogement. PRIEUR, Michel et al. Projet de convention relative au statut international des déplacés environnementaux. In: Revue européenne \begin{tabular}{lllllll}
\hline de droit & de & l'environnement. & n. & 4, & 2008. & Disponível
\end{tabular} <http://www.cidce.org/pdf/Projet\%20de\%20convention\%20relative\%20au\%

20statut\%20international\%20des\%20déplacés\%20environnementaux.pdf>. Acesso em: 20 fev. 2010.

33 La présente Convention a une vocation universelle. Elle porte aussi bien sur les déplaecements envrironnementaux inter-étatiques qu'intra-étatiques.

34 CAVEDON, F. S.; VIEIRA, R. S. Socioambientalismo e Justiça Ambiental. In. BENJAMIN, A. H. ; LECEY, E. ; CAPPELLI, S. (Org.). Mudanças Climáticas, Biodiversidade e Uso Sustentável de Energia. São Paulo : Imprensa Oficial do Estado de São Paulo, 2009.p.559.

35 ISDR (Estratégia Internacional para Redução de Desastres) é um sistema de parceria que auxilia as nações e comunidades na implementação da Declaração de Hyogo (2005) através da vasta participação dos Governos e organizações no ISDR; aumentando o perfil da redução de desastres nas prioridades e programas das organizações, e construindo uma forte e mais sistemática e esforços internacionais coerentes para ajudar na redução dos desastres.

36 Estratégias de redução de desastres e práticas de gerenciamento do risco: elementos críticos para adaptação as mudanças climáticas. 
37. Ensure that disaster risk reduction is a national and a local priority with a strong institutional basis for implementation. 2. Identify, assess and monitor disaster risks and enhance early warning. 3. Use knowledge, innovation and education to build a culture of safety and resilience at all levels. 4. Reduce the underlying risk factors. 5. Strengthen disaster preparedness for effective response. MYERS, Norman. Environmental Refugees: An emergent security issue. Prague: XVIII Economic Forum. 23-27 mai 2005. Disponível em: <http://www.osce.org/ documents/eea/2005/05/14488 en.pdf> Acesso em: 30 mar 2009. HERMSMEYER, Heide A. Environmental Refugees: a denial of rights. International studies Program. University of California. Working Paper n. 2. San Diego, 2005.

40 MYERS, Norman. Environmental Refugees: An emergent security issue. Prague: XVIII Economic Forum. 23-27 mai 2005. Disponível em: <http://www.osce.org/ documents/eea/2005/05/14488 en.pdf> Acesso em: 30 mar 2009.

41 MYERS, Norman. Environmental Refugees: An emergent security issue. Prague: XVIII Economic Forum. 23-27 mai 2005. Disponível em: <http://www.osce.org/ documents/eea/2005/05/14488 en.pdf > Acesso em: 30 mar 2009.

\section{REFERÊNCIAS}

BECK, Ulrich. Risk society towards a new modernity. Londres Sages, 1992.

, Ulrich. La sociedad del riesgo hacia una nueva modernidad. Barcelona Paidós, 1998.

, Ulrich. La sociedad de riesgo global. Trad. Jesús Alborés Rey. Madrid: Siglo

XXI de España, 2002.

BRASIL. Conferência das Nações Unidas sobre Meio Ambiente e Desenvolvimento (1992: Rio de Janeiro). 3. ed. Brasília: Senado Federal, Subsecretaria de Edições Técnicas, 2000.

BROWN, Oli. Migration and climate change. IOM Imigration Research Series, n. 31. Genebra: IOM, 2008.

CASTLES, Stephen. Environmental change and forced migration: making sense of the debate. In: New Issues in Refugee Research. Working Paper n. 70, out 2002. UNHCR (United Nations High Commissioner for Refugees): Genebra, 2002.

CAVEDON, F. S.; VIEIRA, R. S. Socioambientalismo e Justiça Ambiental. In. BENJAMIN, A. H. ; LECEY, E.; CAPPELLI, S. (Org.). Mudanças Climáticas, Biodiversidade e Uso Sustentável de Energia. São Paulo: Imprensa Oficial do Estado de São Paulo, 2009.

COURNIL, Christel. À la recherche d'une protection pour les 'réfugiés environnementaux': actions, obstacles, enjeux et protections. In: Revue Asylon(s), n. 6, nov 2008. Disponível em:

<http://terra.rezo.net/article843.html>. Acesso em: 19 mar 2010.

HERMSMEYER, Heide A. Environmental Refugees: a denial of rights. International studies Program. University of California. Working Paper n. 2. San Diego, 2005.

HUGO, Graeme. Migration, development and environment. IOM Imigration Research Series. n. 35. Genebra: IOM, 2008. 
. Environmental concerns and international migration. In: International migration review. 1996, p. 105-131.

IPCC. Climate Change 2007: Climate change impacts, adaptation and vulnerability summary for policy makers. Contribution of Working Group I to the Fourth Assessment Report of the Intergovernmental Panel on Climate Change, Paris, Abril, 2007.

KLIOT, Nurit. Environmentally induced population movements their complex sources and consequences in KLIOT, Nurit; KROL, Maarten e UNRUH, Jon. Environmental change and it implications for population migration. Norwell, Kluwer Academic Publishers, 2004.

MAGNINY. Véronique. Des victimes de l'environnement aux réfugiés de l'environnement.

In: Revue Asylon(s), n. 6, nov 2008. Disponível em: $<$ http://terra.rezo.net/article845.html>.

Acesso em: 18 março 2010.

MYERS, Norman. Environmental Refugees: An emergent security issue. Prague: XVIII Economic Forum. 23-27 mai 2005. Disponível em: <http://www.osce.org/ documents/eea/2005/05/14488 en.pdf> Acesso em: 30 mar 2009.

, Norman. Environmental Refugees in a globally warmed world. In: BioScience.

Vol. 43, n. 11, dez 1993. pp. 752-761. Disponível em: <http://www.jstor.org/stable/1312319> Acesso em: 17 mar 2009.

PIOVESAN, Flávia. Direitos humanos e o direito constitucional internacional. São Paulo: Max Limonad, 2002.

PRIEUR, Michel et al. Projet de convention relative au statut international des déplacés environnementaux. In: Revue européenne de droit de l'environnement. n. 4, 2008. Disponível em: <http://www.cidce.org/pdf/Projet\%20de\%20convention\%20relative\%20au\% 20statut\%20international\%20des\%20déplacés\%20environnementaux.pdf>. Acesso em: 20 fev. 2010.

TRINDADE, Antônio Augusto Cançado. Direitos Humanos e meio ambiente: paralelo dos sistemas de proteção internacional. Porto Alegre: Fabris, 1993.

WESTRA, Laura. Environmental justice and the rights of ecological refugees. London: Earthscan.2009.

Recebido para publicação: 18.06.2010

Aceito para publicação: 27/12/2010 